\title{
Interval estimation: Effect of processing demands on prospective and retrospective reports
}

\author{
LUCINDA McCLAIN \\ Marquette University, Milwaukee, Wisconsin
}

\begin{abstract}
Undergraduate students performed one of three levels of processing on each word $(15,30$, or 45) presented during a 120-sec interval. Subjects were told in advance that they would be required to estimate the length of the presentation interval (prospective condition) or were presented with an unexpected estimation task (retrospective condition). In the prospective condition, interval estimates were an inverse function of list length when relatively deep levels of processing were required, but were an increasing function of list length when shallow processing was required. In the retrospective condition, estimates were an increasing function of list length and were unaffected by different levels of processing. The interval estimation model proposed by Hicks, Miller, and Kinsbourne (1976) provided a better account of the data than did the storage-size hypothesis of Ornstein (1969).
\end{abstract}

Interval estimation requires subjects to indicate the length of a given interval in the absence of an external timing device. Different paradigms have produced different results and have spawned opposing theories of interval estimation. When subjects have not known in advance that estimates would be required, that is, the retrospective paradigm was used, estimates increased as the amount of information presented during the interval increased (Block, 1974; Mulligan \& Schiffman, 1979; Ornstein, 1969; Underwood \& Swain, 1973). A theory proposed by Ornstein maintains that estimates are directly related to the "storage size" of the interval. Increasing the amount or complexity of the information presented was assumed to increase storage size and thereby lengthen interval estimates.

In studies using the prospective paradigm, that is, subjects knew in advance that estimates would be required, two patterns of results have been reported. In one set of studies (Bakan, 1955; Burnside, 1971; Curton \& Lordahl, 1974; DeWolfe \& Duncan, 1959; Hicks \& Brundige, 1974; McKay, 1977; Vroon, 1970), estimates decreased as the amount of information presented during the interval increased. The second set of studies (Aitken \& Gedye, 1968; Buffardi, 1971; Craig, 1973; Mo, 1975; Thomas \& Brown, 1974) produced the filled-duration illusion-intervals filled with nonverbal stimuli such as lights or tones were

A summary of this paper was presented at the American Psychological Association convention, Washington, D.C., August 1982. $I$ thank Linda Lawrence for assistance in running subjects. Requests for reprints should be sent to Lucinda McClain, Department of Psychology, Marquette University, Milwaukee, Wisconsin 53233. judged longer than "empty" intervals of the same duration. Hicks and his co-workers (Hicks, Miller, \& Kinsbourne, 1976) reconciled this apparent inconsistency by noting that the first group of studies required subjects to actively process information during the interval, while in the second group of studies, information was merely presented.

Although Thomas and Brown (1974) provided a detailed theoretical account of the filled-duration illusion, Hicks (Hicks et al., 1976; Hicks, Miller, Gaes, \& Bierman, 1977; Miller, Hicks, \& Willette, 1978) proposed a model of prospective interval estimation which accounted for both sets of data. Subjective duration is assumed to increase with the subject's attention to time. Attention to time results in the storage of subjective temporal units. These units are based on the average mental content per unit of duration, as suggested by Frankenhaeuser (1959). Mere stimulus presentation does not divert attention from time-in-passing. In this case, increasing the number of stimuli presented during the interval increases judged duration by increasing the number of subjective temporal units stored during that interval. When the stimuli presented during the interval require active processing, less attention can be allocated to time-in-passing, fewer subjective temporal units are stored, and, consequently, judged duration decreases with increased processing demands. In the retrospective paradigm, no subjective temporal units would presumably be stored during the interval because the subject would be allocating no attention to time-in-passing in anticipation of an upcoming estimation task. For this reason, Hicks's model does not provide a direct account of retrospective interval estimation. 
The purpose of the present study was to examine the effects of the same variables on both prospective and retrospective interval estimation. The variables - number of words presented during a fixed interval and level of processing required during the intervalwere selected because of their theoretical significance for both Hicks's and Ornstein's models. Processing during the interval was manipulated by requiring subjects to: (1) graphemically encode the words without instructions to memorize the items (graphemicincidental condition), (2) semantically encode the words without memorization instructions (semanticincidental condition), or (3) semantically encode the words and store them for subsequent recall (semantic-intentional condition). Conditions $1-3$ required progressively deeper levels of processing (Craik \& Lockhart, 1972), Graphemic encoding, rather than simple item presentation, was used to help ensure that subjects attended to all stimuli at each level of processing. Subjects were tested for recall of words presented during the interval in an attempt to measure storage size.

Increasing the number of words presented during the interval and the level of processing required were both expected to increase storage size as measured by the number of words correctly recalled. Ornstein's (1969) theory predicted a direct relationship between interval estimates and storage size in the retrospective and prospective conditions. Although Ornstein had tested his theory in the retrospective paradigm, other investigators (Schiffman \& Bobko, 1974, 1977) had used his model to account for results obtained in the prospective paradigm.

Hicks's (Hicks et al., 1976; Hicks et al., 1977; Miller et al., 1978) theory predicted that interval estimates would be determined by the amount of processing required during the interval. When relatively deep processing was required (semantic encoding conditions), estimates were expected to be an inverse function of the number of words presented. If shallow processing (graphemic encoding condition) was the functional equivalent of mere stimulus presentation, estimates would be expected to be a direct function of the number of words presented. These predictions were made for prospective interval estimates. The effects of these variables on retrospective estimates could not be anticipated from Hicks's model. It should be noted that Hicks (Hicks et al., 1976; Miller et al., 1978) found no effect of processing demands on retrospective estimates in conditions most similar to those used in the present study.

\section{METHOD}

\section{Subjects}

The subjects were 180 undergraduates (111 females) enrolled in an introductory psychology course. Extracredit points were awarded for their participation in the 20-min session. Each student was run individually.

\section{Materials and Apparatus}

From a pool of 46 common English nouns, lists of 15, 30, and 45 items were prepared. In each list, half the nouns were "living" (e.g., CAT, FRIEND, BEAN) and half were "nonliving" (e.g., SWEAT, EVENING, STEP). Living and nonliving items were matched for natural language frequency (Kučera \& Francis, 1967). In addition, half the words in each list began with the letters A-L and half began with the letters M-Z. All words contained three to seven letters.

Slides of the words were individually presented on a rearprojection screen. At a viewing distance of $1 \mathrm{~m}$, the words subtended average visual angles of $4.1 \mathrm{deg}$ horizontally and $1.3 \mathrm{deg}$ vertically. Presentation rate was controlled by a clockcounter in combination with a tachistoscopic shutter.

\section{Procedure}

In a 120 -sec interval, 15,30 , or 45 words were presented at rates of one item every 8,4 , or $2.67 \mathrm{sec}$, respectively. For all presentation rates, the interstimulus interval was $.6 \mathrm{sec}$. In the graphemic encoding condition, subjects were required to classify each word as beginning with the letters $A-L$ or $M-Z$ by pressing one of two buttons mounted on the table in front of them. In the semantic encoding conditions, subjects classified each word as living or nonliving using the same buttons. Appropriate button labels were used for each encoding condition. Students in the semanticincidental and graphemic-incidental conditions received only the classification instructions. In the semantic-intentional condition, the students were also instructed to remember each word for a recall test. Classification decisions were manually recorded by the experimenter.

In the prospective conditions, students were informed before list presentation that they would subsequently have to estimate the length of the interval in which the words were presented. In the retrospective conditions, students were not informed of this fact. Estimation instruction (prospective or retrospective), list length $(15,30$, or 45 words), and level of processing required (semantic-intentional, semantic-incidental, or graphemic-incidental) were between-subject variables. Ten students were randomly assigned to each of the 18 conditions. In each condition, the word list was presented in two random orders, with half the students receiving each order.

After receiving the appropriate instructions, students were asked to remove their watches and jewelry because "these metal objects sometimes interfere with our electronic recording equipment." The students were also questioned several times about their prior knowledge of the experimental procedure; 43 students were replaced because they admitted they had discussed the experiment with students who had previously participated. Students in the prospective conditions were instructed not to count or otherwise mark time during presentation of the word lists.

Immediately after list presentation, subjects in all conditions estimated the 120-sec interval using the reproduction method. Reproduction, rather than verbal estimation or magnitude estimation, was used because it had been suggested (McKay, 1977) that this measurement technique was more sensitive. The word TIME appeared on the screen, and the subjects were instructed to press one of the buttons when an interval had elapsed which was equal to the interval in which the words had been presented. The experimenter manually recorded, to the nearest $.01 \mathrm{sec}$, the interval estimate as measured by the clockcounter. Approximately 1 min was required to instruct all subjects on how to make their interval estimates.

A 4-min free recall test followed the estimation task. The subjects then repeated the interval estimation. During the first estimation, subjects in the semantic-intentional condition were presumably rehearsing for the upcoming recall test, whereas subjects 
in the incidental conditions were not rehearsing. It was possible that different amounts of concurrent rehearsal would affect interval estimates, as Miller et al. (1978) had reported.

\section{RESULTS}

\section{Classification}

Subjects performed the classification task during list presentation with high accuracy. In all retrospective conditions, accuracy was $>97 \%$. In the prospective paradigm, classification decisions in the semanticintentional condition were $93 \%$ correct for the 45 item list. In the remaining prospective conditions, accuracy was $>96 \%$.

\section{Recall}

The number of words correctly recalled was analyzed with an estimation instruction $\times$ list length $\times$ levels of processing analysis of variance. The main effects of list length $[F(2,162)=78.81, p<.01]$ and level of processing $[F(2,162)=205.98, p<.01]$ were significant, as was the list length $\times$ levels of processing interaction $[\mathrm{F}(4,162)=7.85, \mathrm{MSe}=7.85, \mathrm{p}<.01]$. The mean numbers of words correctly recalled from the 15-, 30-, and 45-item lists were $10.00,12.65$, and 18.85 in the semantic-intentional condition, 7.00, 10.20 , and 16.20 in the semantic-incidental condition, and $3.40,3.75$, and 4.25 in the graphemicincidental condition. A critical difference of 2.28 words was required for statistical significance $(p<$ $.01)$ in comparisons of these means. The levels-ofprocessing variable affected the number of words recalled. At each list length, subjects in the semanticintentional condition recalled significantly more words than the subjects in the semantic-incidental condition, who, in turn, recalled significantly more words than the subjects in the graphemic-incidental condition.

In the graphemic encoding condition, which required relatively superficial processing, list length did not significantly affect the number of words correctly recalled. This result is consistent with previous findings (cf. Cooper \& Pantle, 1967) that, under certain conditions, recall depends on the length of the presentation interval and not on the number of items presented. Deeper levels of processing were required in the semantic encoding conditions. In both these conditions, increases in list length produced significant increases in the number of words recalled. The main effect of estimation instruction and all other interactions were not significant.

\section{Interval Estimation}

To determine whether the two interval estimates differed as a function of estimation instruction, list length, and level of processing required, a four-factor analysis of variance was conducted. First- and secondinterval estimate was a within-subjects variable; the remaining factors were between-subjects variables. The two estimates did not significantly differ $[F(1,162)$ $=1.75, p>.05]$, and this variable did not enter into any interactions.

Estimates from the first reproduction task were then analyzed with an estimation instruction $\times$ list length $\times$ levels of processing analysis of variance. The main effects of list length and levels of processing, as well as the estimation instruction $\times$ list length, estimation instruction $\times$ levels of processing, and list length $\times$ levels of processing interactions, were significant $(p<.01)$. These effects can best be understood with reference to the significant estimation instruction $\times$ list length $\times$ levels of processing interaction $[F(4,162)=3.77, \quad M S e=1018.61, p<.01]$ shown in Figure 1. Note that total presentation time was the same for all list lengths because the longer lists were presented more rapidly.

In the prospective paradigm, the list length $x$ levels of processing interaction was significant $[F(4,162)$ $=7.31, \mathrm{p}<.01$ ], while in the retrospective paradigm, this interaction was not significant $[F(4,162)<1]$. When prospective estimates were required, interval estimates were an inverse function of list length in both semantic encoding conditions. Interval estimates were a direct function of list length in the graphemic encoding condition. In all three processing conditions, the estimates following the 45 -item list significantly differed from estimates following the 15-item list. For all list lengths, estimates in the semantic-intentional condition were significantly shorter than estimates in the semantic incidental con-

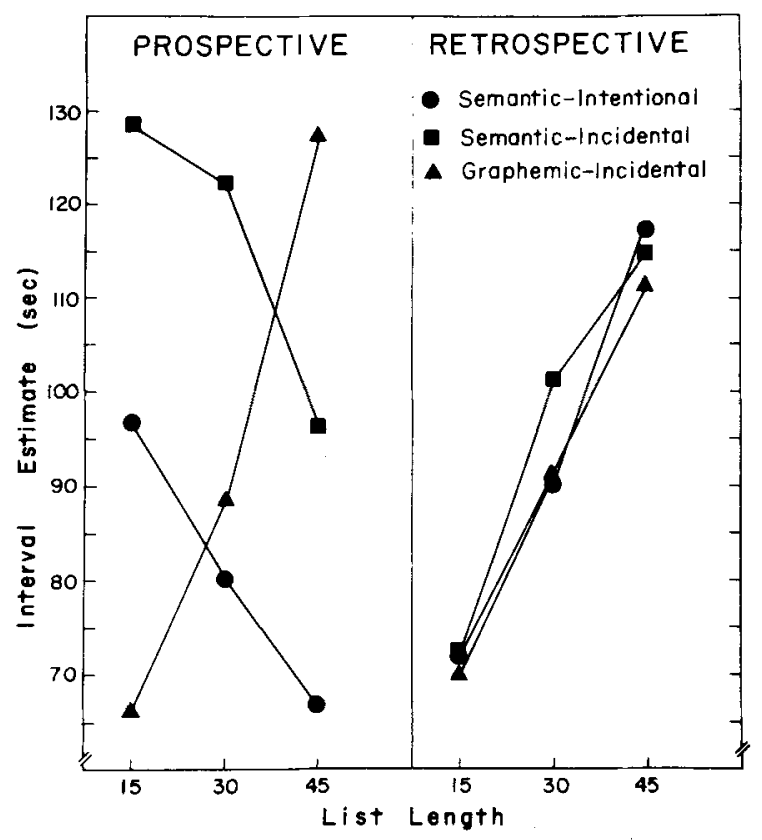

Figure 1. Prospective and retrospective interval entimates (seconds) in three processing conditions as a function of list length. 
dition. A critical difference of $27.97 \mathrm{sec}$ was required for statistical significance $(p<.05)$ in a comparison of means, as shown in Figure 1.

When retrospective estimates were required, interval estimates were a direct function of list length in all processing conditions. None of the differences between processing conditions exceeded the critical value of $27.97 \mathrm{sec}$. In each processing condition, estimates following the 45 -item list were significantly longer than estimates following the 15-item list.

\section{DISCUSSION}

The purpose of the present study was to test predictions from Hicks's (Hicks et al, 1976; Hicks et al., 1977; Miller et al., 1978) and Ornstein's (1969) theories of interval estimation in both the prospective and retrospective paradigms. The adequacy of each model in accounting for the observed data will be considered.

According to Ornstein's model, interval estimates depend on the storage size of the interval. Although Ornstein stated that storage size could never really be measured, storage size was assumed to increase as the number of events stored and retrieved increased. If storage size is operationally defined as the number of items presented, some aspects of the present data are in accordance with the predictions of Ornstein's model. In all retrospective conditions and in the graphemic prospective condition, interval estimates increased as list length increased.

The number of words recalled, rather than the number of words presented, seems a more exacting measure of storage and retrieval. If storage size is defined as the number of words recalled, Ornstein's model does not account for the present data. The levels-of-processing variable influenced the number of words recalled at all list lengths. More words were recalled in the semantic-intentional condition than in the semantic-incidental condition, yet interval estimates in these two semantic conditions did not differ in the retrospective paradigm, and were negatively related to recall in the prospective paradigm. In addition, in both the prospective and retrospective paradigms, interval estimates in the graphemic condition increased as a function of list length, although recall from these lists remained constant. Using retrospective paradigms, Block (1974) also found that interval estimates were directly related to the number of words presented, but not to the number of words recalled, and Block and Reid (1978) reported that a levels-of-processing variable did not affect interval estimates.

According to Hicks's model, interval estimates should depend on the amount of information processing required during the interval. The results obtained in the prospective conditions of the present study are generally in accordance with the predictions of this model and in agreement with previous studies using the prospective paradigm (Curton \& Lordahl, 1974; Hicks \& Brundige, 1974; Hicks et al., 1976; Hicks et al., 1977; McKay, 1977; Miller et al., 1978). In the semantic encoding conditions, where relatively deep levels of processing were required, interval estimates were an inverse function of list length. The semantic-intentional condition presumably required more processing than the semantic-incidental condition, and the semantic-intentional condition produced shorter estimates at all list lengths. The combined effects of level of processing and list length may be referred to as the processing demand. In terms of Hick's model, as processing demands increased, fewer subjective temporal units could be stored during the interval. Consequently, as processing demands increased, interval estimates decreased.

In the graphemic encoding condition, relatively shallow processing was required during the interval. According to Hicks's model, with minimal processing, more subjective temporal units would be stored as list length increased. As predicted, interval estimates in the graphemic condition were an increasing function of list length. It is noteworthy that the increasing function obtained in the present graphemic condition is similar to the results of previous prospective studies (Buffardi, 1971; Craig, 1973; Mo, 1975; Thomas \& Brown, 1974), which used nonverbal stimuli and required no active processing during the interval.

According to Hicks's model, the graphemic condition provided a greater opportunity for the creation of subjective temporal units than did the semantic conditions. If more subjective temporal units were created in the graphemic condition, interval estimates should be longer in that condition than in the semantic conditions. The average estimate in the graphemic condition $(94.02 \mathrm{sec})$ was significantly $(p<.01)$ shorter than the estimate in the semantic-incidental condition $(115.56 \mathrm{sec})$ and did not differ significantly from the estimate in the semantic-intentional condition $(81.26 \mathrm{sec})$.

Hicks's model could account for this result if we distinguish the opportunity to create subjective temporal units and their actual creation. The creation of one subjective temporal unit requires a certain amount of mental content, and the mental content generated by one classification decision in the graphemic condition was presumably less than in the semantic conditions. Subjects in the graphemic condition had more opportunity to create subjective temporal units, but the low mental content generated by the task led to the creation of relatively few. Subjects in the semantic conditions had less opportunity to create subjective temporal units (due to the demands of the task), but sufficient mental content to support their creation whenever the opportunity arose. This account does not predict longer estimates in shallow 
processing conditions because of a tradeoff between the opportunity to create subjective temporal units and their actual creation.

In the retrospective conditions, interval estimates were an increasing function of list length and were unaffected by the level of processing required. Hick's model could account for these results if we assume that retrospective interval estimates are basically a reconstructive process, that is, subjective temporal units can be created and stored after the interval has elapsed. Rather than assuming that this reconstruction is based on memory for the information presented during the interval as Ornstein has suggested, this reconstructive process could be based on memory of the number of processing decisions made during the interval. In the present study, more decisions were made during the 120 -sec interval as list length increased and the same number of classification decisions were made in each levels-of-processing condition. This account could explain how retrospective estimates were unaffected by the level of processing required during the interval.

The present study found that processing demands differentially affected prospective and retrospective interval estimation. Hicks's model of interval estimation provided a better general account of these results than did Ornstein's model. The present data do not readily suggest an alternative theoretical explanation, nor do they appear to be consistent with the model proposed by Hogan (1978) to reconcile discrepancies between theories like those of Hicks and Ornstein. Future research should be directed toward development of a model that can more specifically account for both prospective and retrospective interval estimation.

\section{REFERENCES}

Aitken, R.B.C., \& GeDYe, J. L. A study of two factors which affect arousal level and the apparent duration of a ten-minute interval. British Journal of Psychology, 1968, 59, 253-263.

BaKan, P. Effect of set and work speed on time estimation. Perceptual and Motor Skills, 1955, 5, 147-148.

BLock, R. A. Memory and the experience of duration in retrospect. Memory \& Cognition, 1974, 2, 153-160.

Brock, R. A., \& Reid, M. A. Remembered duration: Evidence for a contextual-change hypothesis. Journal of Experimental Psychology: Human Learning and Memory, 1978, 4, 656-665.

Buffard, L. Factors affecting the filled-duration illusion in the auditory, tactual, and visual modalities. Perception \& Psychophysics, 1971, 10, 292-294.

Bunnside, $W$. Judgment of short time intervals while performing mathematical tasks. Perception \& Psychophysics, 1971, 9, 404-406.
Cooper, E. H., \& Pantle, A. J. The total-time hypothesis in verbal learning. Psychological Bulletin, 1967, 68, 221-234.

Craig, J. C. The constant error in the perception of brief temporal intervals. Perception \& Psychophysics, 1973, 13, 99-104.

Craik, F.I.M., \& Lockhart, R. C. Levels of processing: A framework for memory research. Journal of Verbal Learning and Verbal Behavior, 1972, 11, 671-674.

Curton, E. D., \& Lordahl, D. S. Effects of attentional focus and arousal on time estimation. Journal of Experimental Psychology, 1974, 103, 861-867.

DeWolfe, R. K., \& Duncan, C. P. Time estimation as a function of level of behavior of successive tasks. Journal of Experimental Psychology, 1959, 58, 153-158.

Frankenhaeuser, M. The psychology of time. Stockholm: Almqvist \& Wiksell, 1959.

Hrcks, R. E., \& Brundige, R. M. Judgments of temporal duration while processing verbal and physiognomic stimuli. Acta Psychologica, 1974, 38, 447-453.

Hicks, R. E., Miller, G. W., Gaes, G., \& Bierman, K. Concurrent processing demands and the experience of time-inpassing. American Journal of Psychology, 1977, 90, 431-446.

Hicks, R. E., Miller, G. W., \& Kinsbounne, M. Prospective and retrospective judgments of time as a function of amount of information processed. American Journal of Psychology, 1976, 89, 719-730.

Hogan, H. W. A theoretical reconciliation of competing views of time perception. American Journal of Psychology, 1978, 91, 417-428.

Kučera, H., \& Francis, W. N. Computational analysis of present-day American English. Providence, R.I: Brown University Press, 1967.

McKAY, T. D. Time estimation: Effects of attentional focus and a comparison of interval conditions. Perceptual and Motor Skills, 1977, 45, 584-586.

Miller, G. W., Hicks, R. E., \& Willette, M. Effects of concurrent verbal rehearsal and temporal set upon judgments of temporal duration. Acta Psychologica, 1978, 42, 173-179.

Mo, S. S. Temporal reproduction of duration as a function of numerosity. Bulletin of the Psychonomic Society, 1975, 5, 165-167.

Mulligan, R. M., \& Schiffman, H. R. Temporal experience as a function of organization in memory. Bulletin of the Psychonomic Society, 1979, 14, 417-420.

Onnstein, R. E. On the experience of time. Middlesex, England: Penguin, 1969.

Schiffman, H. R., \& Boвко, D. J. Effects of stimulus complexity on the perception of brief temporal intervals. Journal of Experimental Psychology, 1974, 103, 156-159.

Schifrman, H. R., \& Boвko, D. J. The role of number and familiarity of stimuli in the perception of brief temporal intervals. American Journal of Psychology, 1977, 90, 85-93.

Thomas, E.A.C., \& Brown, I., JR. Time perception and the filled-duration illusion. Perception \& Psychophysics, 1974, 16, 449-458.

Underwood, G., \& Swain, R. A. Selectivity of attention and the perception of duration. Perception, 1973, 2, 101-105.

Vroon, P. A. Effects of presented and processed information on duration experience. Acta Psychologica, 1970, 34, 115-121.

(Manuscript received September 7, 1982; revision accepted for publication March 23, 1983.) 